\title{
The Effect of a Single Passive Intervention to Improve Patient Satisfaction in an Orthopaedic Service
}

\author{
Manit Arora $^{1 *}$, Ian A. Harris ${ }^{1,2}$, Lynette McEvoy ${ }^{2}$, Rajat Mittal ${ }^{1,2}$, Justine M. Naylor ${ }^{2}$ \\ ${ }^{1}$ South Western Sydney Clinical School, University of New South Wales, Sydney, Australia; ${ }^{2}$ Whitlam Orthopaedic Research Centre, \\ Liverpool Hospital, Sydney, Australia. \\ Email: *manit_arora@hotmail.com
}

Received December $13^{\text {th }}, 2011$; revised February $8^{\text {th }}, 2012$; accepted March $10^{\text {th }}, 2012$

\begin{abstract}
Patient satisfaction is a goal of effective health care delivery and its assessment is important to the improvement of health care, especially in the context of a more consumerist culture. Patient education and information has been shown to improve patient satisfaction with care. Lack of sufficient patient information (specifically related to post-discharge care) was identified in our patient population by means of a broad ranging post-discharge satisfaction survey. Targeted intervention, in the form of a discharge information sheet for patients, was trialled with the aim of improving specific satisfaction parameters related to post-discharge care, and overall patient satisfaction. Patient satisfaction was measured for six months before and after the intervention, and data from both groups compared. There was no statistically significant difference in patient satisfaction directly related to the provision of the additional discharge information or overall patient satisfaction between the two groups. Providing patients with specific information alone at discharge is not sufficient to improve overall or discharge-specific patient satisfaction. More active interventions may be required.
\end{abstract}

Keywords: Orthopaedics; Patient Satisfaction; Intervention

\section{Introduction}

High levels of patient satisfaction should be an important outcome of all surgical care, with greater patient satisfaction associated with superior compliance, improved attendance at follow-up visits and better outcomes [1]. Assessment of patient satisfaction is an important measure of the quality of care because it reflects how well health care providers meet the expectations that are most relevant to the patient [2]. Clinicians are poor judges of patient satisfaction, especially when patients are dissatisfied [3], and accordingly validated questionnaires have been developed to assess patient satisfaction.

There are many models that have been designed to capture the drivers of patient satisfaction. Traditionally, these drivers were thought to be patient expectation, patient characteristics and psycho-social determinants. These traditional models have been refined by further research. Patient characteristics are, at best, a minor predictor of patient satisfaction [4]. Previous studies have found that older age, lower level of education, being married and higher social statuses are associated with greater patient satisfaction [2]. However, the strengths of these relationships are poor, and results from various studies are

${ }^{*}$ Corresponding author. often contradictory, except for older age which is uniformly associated with greater patient satisfaction. Patient expectations play a significant role- The more a doctor's performance meets patient expectations, the more satisfied a patient will be with the doctor's services [5]. Dissatisfaction tends to be only expressed when an extremely negative event occurs [6], exemplifying the importance of psycho-social determinants.

Sitiza and Wood (1997) analysed 195 papers on patient satisfaction to determine the factors most influential in shaping satisfaction with healthcare services (Figure 1). Interpersonal aspects of care (communication and empathy) were identified as the most important [7]. Patient education and information were also identified as integral drivers of patient satisfaction. The provision of information by doctors has been found to be positively related to patient satisfaction [8]. A meta-analysis of relevant literature [9] found that the higher the amount of information provided by the doctor, the more positive the impact this had on patient satisfaction. A limitation of this particular study is that most studies had varied methods of analysing patient satisfaction; this could be improved by using a standard validated satisfaction questionnaire for each study.

Many orthopaedic procedures require patients to adhere 


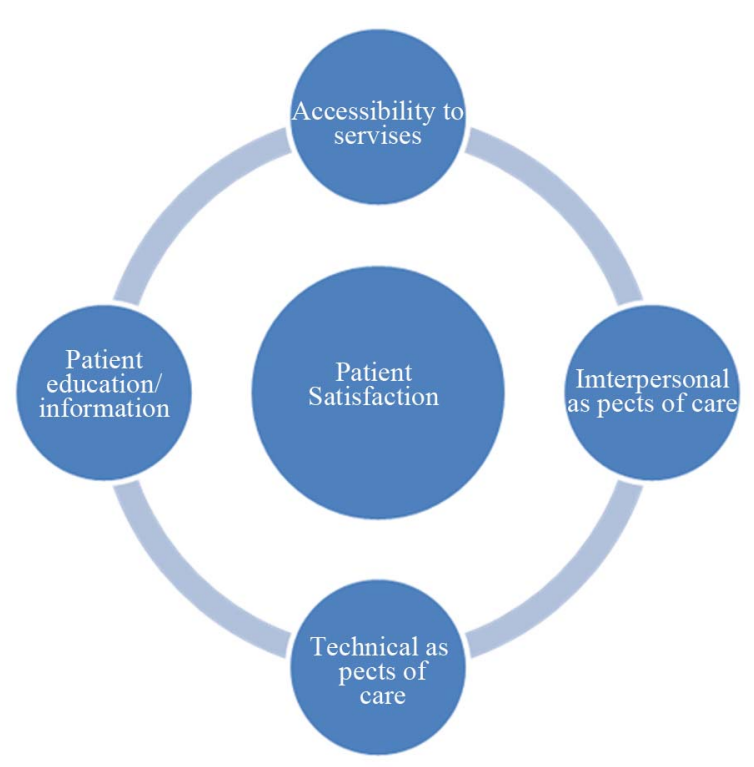

Figure 1. The components of patient satisfaction (data modified from: (Sitiza \& Wood, 1997)).

to specific protocols and/or participate in regimented rehabilitative or follow-up procedures. In this regards, patient satisfaction is not only important alone, but also serves the multi-purpose of promoting greater compliance, improved attendance at follow up visits and better outcomes with these aspects of care, as described above. Despite this, there has been very little work on patient satisfaction in orthopaedics. As a preface to a larger study designed to improve patient engagement with care in orthopaedic practice, this study aimed to determine patient satisfaction with current standards of care and to address areas of deficiency in this care. Through a broad ranging post-discharge satisfaction survey we identified that patients had relatively low rates of satisfaction regarding discharge instructions. We hypothesized that providing patients with education and information in the form of a targeted intervention (discharge information sheet) will improve patient satisfaction, particularly satisfaction related to the provision of discharge information.

\section{Methodology}

We conducted a prospective single centre cohort study of patients presenting to the Orthopaedic Department at our hospital. Inclusion criteria included all adult patients discharged from the Orthopaedic ward between January and December 2010. Exclusion criteria included patients residing in nursing homes and those referred to ongoing in-patient rehabilitation. Patients were divided into two groups: January-June 2010 (non-intervention group) and July-December 2010 (intervention group). All patients were contacted by mail two weeks after discharge and asked to complete a comprehensive satisfaction survey.
Non-responders were contacted by telephone within two weeks of the date of mailing and asked to complete the mailed survey, or to complete the same survey via telephone.

The self-administered questionnaire is a modified version of the validated New South Wales (NSW) Health Questionnaire [10], which is administered on a yearly basis to a sample of patients who presented to all NSW public hospitals. Our questionnaire contained a subset of questions ( $n=35$ ) used in the Health Questionnaire, and was designed to assess certain parameters of orthopaedic patient satisfaction. Questions ranged from investigating demographic profile of patients to level of content with nursing, medical and allied health staff. This broad ranging survey encompassed aspects of both inpatient and post-discharge care. Two questions specifically related to discharge information were: "Were you told if you needed physiotherapy and when to commence physiotherapy?" (Answer: Yes/No/Not applicable) and "Did you know who to call if you needed help or had more questions after you left?” (Answer: Yes/No). The questions on overall satisfaction were: "Overall, how would you rate the care you received at the hospital?” (Answer: Poor/Fair/ Good/Very Good/Excellent) and an overall rating of hospital from best to worst possible (0 - 10 scale).

The intervention was designed on the basis of our baseline survey. This baseline survey is the same validated proforma as our study survey and was distributed to patients between July and December 2009. Based on the results of this data, we identified poor scores (relative to other questions) regarding post-discharge care such as when to commence physiotherapy and who to contact for help. Accordingly, we designed a targeted intervention in the form of a discharge information sheet that specifically contained this information. This was distributed to all patients (Orthopaedics Ward) at time of discharge. They were written in English only and were not accompanied by any oral instructions or other multimedia formats.

Data were analysed using SAS (Cary, NC, USA). Data relating to the provision of discharge information and overall satisfaction were compared using the chi-square test. Data on overall hospital rating (using numerical rating scale) were compared using Student's $t$-test (SAS system). Statistical significance was defined as a $P$-value less than 0.05 .

\section{Results}

The non-interventional group (January-June 2010) had 97 responses (280 surveys sent; 35\% response rate) and the interventional group (July-December 2010) had 107 responses (240 surveys sent; 45\% response rate). Demographic profile showed $50 \%$ of our responders had an education level less than high school diploma and $49 \%$ were born overseas. The parameters studied include: 
1) "Did you know who to call if you needed help or had more questions after you left?" The non-intervenetional group $(n=94)$ had a positive response frequency of $63(67 \%)$. The interventional group $(n=104)$ had a positive response frequency of $72(69 \%) . P$ value $=0.74$.

2) "Were you told if you needed physiotherapy and when to commence physiotherapy?” The non-intervenetional group $(n=97)$ had a positive response frequency of $64(66 \%)$. The interventional group $(n=106)$ had a positive response frequency of $72(68 \%)$. $P$ value $=0.94$.

3) Overall Patient Satisfaction. The non-intervenetional group $(n=97)$ had an overall mean rating of hospital care of 7.1 (Standard deviation = 2.28). The interventional group $(n=107)$ had an overall mean rating of hospital care of 7.4 (Standard deviation $=2.2) . P$ value $=$ 0.61 (Figure 2).

\section{Discussion}

Patient satisfaction is a dynamic field driven by greater consumer awareness and expectations. Despite this, there is a substantial void of research into patient satisfaction in orthopaedics. Our study aimed to improve discharge specific parameters and overall patient satisfaction using a passive intervention (discharge information sheets). We hypothesized that the provision of the targeted intervenetion would improve deficiencies in post-discharge care (as highlighted by the baseline survey), and hence improve overall patient satisfaction. The introduction of the intervention had no significant impact on discharge specific satisfaction parameters or overall patient satisfaction. This outcome is in contrast to previous literature in the field of patient satisfaction showing that the provision of written information to patients should improve patient satisfaction [8]. Additionally, models centre on the importance of patient education and information in improving patient satisfaction (Figure 1).

There are several possible explanations for our failure to significantly improve satisfaction. Firstly, pre-intervention satisfaction levels in our population were not low,

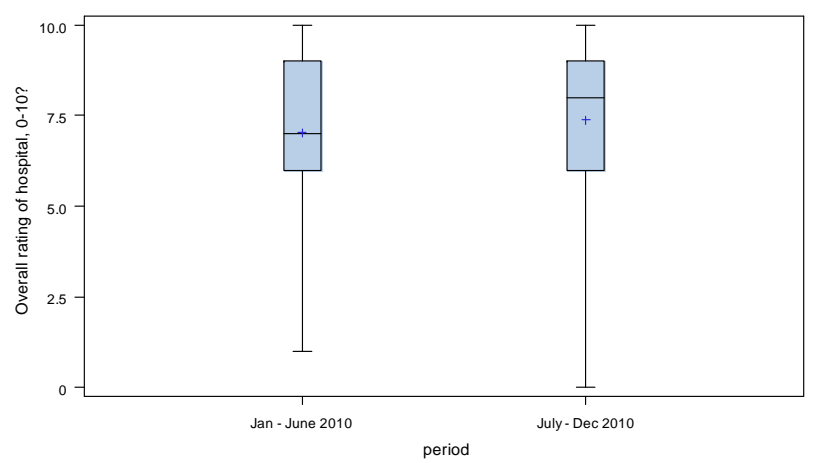

Figure 2. Box and Whisker plot of overall patient satisfaction for non-interventional (Jan-June 2010) and intervenetional (July-Dec 2010) groups, as compared by numerical rating scale (left hand axis). and, at best, a modest impact on overall satisfaction would have been observed. However, we still expected a direct impact on the target of the intervention-post-discharge parameters.

Effective delivery of the intervention may have been affected due to the demographic profile of our population. A significant proportion of our patients are from nonEnglish speaking backgrounds and/or of low socio-economic status. Consequently, patients may not be able to understand the information provided to them or complete the survey effectively. This may be improved through the use of multi-lingual information sheets and surveys. Additionally, a change in delivery format of the information may be required, such as an educational video containing discharge specific information that could be shown to patients at discharge and a copy of this same video in disc format and written format be provided to the patient.

The low response rate may have been a cause of the lack of significance, if patients who were more likely to have responded to the discharge information were less likely to respond. We attempted to improve the response rate through phone calls to the responders encouraging return of completed survey or the option to complete the survey via phone. It is possible that additional corrective measures may have improved the response rate, such as arranging for courier pick up of surveys or creating a monetary reward for successful participation. The latter may be particularly useful in the context of our low socio-economic demographic profile.

Ingratiating response bias [7], where the patient uses the satisfaction survey to ingratiate themselves with researchers or medical staff- and self interest bias, where patients judge that their expressions of satisfaction will contribute to the continuation of the health service which in turn will be in their own self-interest-may complicate data. Corrective measures here include disclaimers stating that the survey has no impact on future admissions or the management of the current condition.

There has been minimal research in the field of patient satisfaction in Orthopaedics. Most research has focused on assessing patient satisfaction after specific procedures such as total hip arthroplasty or total knee replacement. Such studies aim to assess patient satisfaction from a surgical point of view, with critical analysis of the relation of technical aspects of care to patient satisfaction. Improving patient satisfaction requires a multifaceted approach addressing, but not limited to, accessibility to services, interpersonal aspects of care and technical aspects of care, as well as patient education and information (Figure 1). Consequently, it is possible that the provision of discharge information sheets needs to be supplemented with improvement in these areas in order to affect overall patient satisfaction. 


\section{Conclusion}

Patient education and information is an integral driver of patient satisfaction and should be an important component of healthcare delivery. However, the provision of information should be validated for effectiveness. We have shown that the provision of discharge information sheets as an intervention to improve both patient satisfaction regarding certain aspects of post-discharge care, and overall patient satisfaction, was not effective. We recommend trialling a more active form of this information, as described above, along with supplementary material and adjustments to delivery format.

\section{REFERENCES}

[1] P. D. Cleary, et al., "Patient Assessments of Hospital Care," Quality Review Bulletin, Vol. 15, No. 6, 1989, pp. 172-179.

[2] P. L. N. Thi, et al., "Factors Determining Inpatient Satisfaction with Care,” Social Science and Medicine, Vol. 54, No. 4, 2002, pp. 493-504.

[3] H. R. Rubin, "Patient Evaluations of Hospital Care: A Review of the Literature," Medical Care, Vol. 28, No. 9, 1990, pp. 3-9.

[4] J. Hall and M. Dornan, "Patient Sociodemographic Char- acteristics as Predictors of Satisfaction with Medical Care: A Meta-Analysis,” Social Science and Medicine, Vol. 30, No. 7, 1990, pp. 811-818.

[5] D. Mechanic, "Medical Sociology,” Free Press, New York, 1968

[6] B. Williams, "Patient Satisfaction: A Valid Concept?" Social Science and Medicine, Vol. 38, No. 4, 1994, pp. 509-516.

[7] J. Sitiza and N. Wood, "Patient Satisfaction: A Review of Issues and Concepts,” Social Science and Medicine, Vol. 45, No. 12, 1997, pp. 1829-1843.

[8] S. Williams, J. Weinmann and J. Dale, "Doctor-Patient Communication and Patient Satisfaction: A Review," Family Practice, Vol. 15, No. 5, 1998, pp. 480-492.

[9] D. Roter, J. Hall and N. Katz, "Patient-Physician Communication: A Descriptive Summary of the Literature," Patient Education and Counselling, Vol. 12, No. 2, 1988, pp. 99-119.

[10] Health, New South Wales (NSW Health), "NSW Health Hospital Care Overnight Patient Survey," 2009. www.health.nsw.gov.au/pubs/2009/pdf/patient_survey_2 009.pdf

[11] S. Abramowitz, A. Cote and E. Berry, "Analyzing Patient Satisfaction: A Multianalytic Approach,” Quality Review Bulletin, Vol. 13, No. 4, 1987, pp. 122-130. 